\title{
ON PSEUDOMONOTONE SET-VALUED MAPPINGS IN TOPOLOGICAL VECTOR SPACES
}

\author{
A. P. FARAJZADEH ${ }^{1}$
}

(Received 21 August, 2008; revised 15 November, 2008)

\begin{abstract}
In this paper we extend results of Inoan and Kolumban on pseudomonotone set-valued mappings to topological vector spaces. An application is made to a variational inequality problem.
\end{abstract}

2000 Mathematics subject classification: primary 49J40; secondary 90C33.

Keywords and phrases: upper semi-continuity, C-pseudomonotonicity, KnasterKuratowski-Mazurkiewicz mapping.

\section{Introduction}

Different notions of pseudomonotonicity are used in functional analysis in the context of nonlinear operators. They are important in the theory of variational inequalities, in optimization, and equilibrium problems (see $[1,2,6]$ ). In many applications concerning elastic-plastic torsion problems, contact problems, heat conduction, thermoelasticity and economic theory there appear nonmonotone, possibly multivalued maps (see for instance $[9,10]$ ).

In 1968, Brezis [2] introduced one type of pseudomonotone operator which was named by many authors as a topological pseudomonotone operator. Another type of pseudomonotone operator was introduced by Karamardian [8] in 1976 in the single-valued case. This pseudomonotonicity notion is sometimes called algebraic. These two pseudomonotonicity concepts are different (see [7]). Inoan and Kolumban [7] studied three types of pseudomonotone set-valued mappings in a topological vector spaces setting. Two of them are generalizations of the classical notions mentioned above. The third generalization, which they called $C$-pseudomonotonicity, is a weaker notion and common generalization of the algebraic and topological pseudomonotonicity for set-valued maps. This paper is inspired and

\footnotetext{
${ }^{1}$ Department of Mathematics, Razi University, Kermanshah, 67149, Iran; e-mail: faraj1348@yahoo.com, ali-ff@sci.razi.ac.ir.

(C) Australian Mathematical Society 2009, Serial-fee code 1446-1811/2009 \$16.00
} 
motivated by [7]. In this paper we generalize the main results of [7] to the topological vector spaces.

\section{Pseudomonotone set-valued mappings}

Throughout the paper, let $X$ and $Y$ be two real Hausdorff topological vector spaces, $T: X \rightarrow 2^{Y}$ be a set-valued mapping, and $\langle\cdot, \cdot\rangle: X \times Y \rightarrow \mathbb{R}$ be a bilinear continuous function.

Definition 2.1 (Inoan and Kolumban [7]). The mapping $T$ is called $A$-pseudomonotone if, for every $x, x^{\prime} \in X$,

$$
\sup _{f \in T(x)}\left\langle x^{\prime}-x, f\right\rangle \geq 0 \quad \text { implies } \sup _{f \in T\left(x^{\prime}\right)}\left\langle x^{\prime}-x, f\right\rangle \geq 0 .
$$

Definition 2.1 generalizes the notion of algebraic pseudomonotonicity, which was introduced by Karamardian [8], for set-valued mappings. The following definition generalizes the notion of topological pseudomonotonicity introduced by Brezis.

Definition 2.2 (Inoan and Kolumban [7]). The mapping $T$ is called B-pseudomonotone if, for every $x \in X$ and for every net $\left\{x_{i}\right\}_{i \in I}$ in $X$ with $x_{i} \rightarrow x, f_{i} \in T\left(x_{i}\right)$, and $\liminf _{i}\left\langle x-x_{i}, f_{i}\right\rangle \geq 0$, implies that for every $y \in X$ there exists $f(y) \in T(x)$ such that

$$
\limsup _{i}\left\langle y-x_{i}, f_{i}\right\rangle \leq\langle y-x, f(y)\rangle .
$$

Definition 2.3 (Inoan and Kolumban [7]). We say that $T$ is $C$-pseudomonotone if, for every $x, y \in X$ and every net $\left\{x_{i}\right\}$ in $X$ with $x_{i} \rightarrow x$,

$$
\begin{aligned}
& \sup _{f \in T\left(x_{i}\right)}\left\langle(1-t) x+t y-x_{i}, f\right\rangle \geq 0, \quad \forall t \in[0,1], \forall i \in I, \\
& \quad \Rightarrow \sup _{f \in T(x)}\langle y-x, f\rangle \geq 0 .
\end{aligned}
$$

REMARK 2.4. We make the following remarks.

(a) There are some examples of applications which are $C$-pseudomonotone but are neither $A$-pseudomonotone nor $B$-pseudomonotone (see [7, Example 5]).

(b) We can define the above definitions on a nonempty convex subset of $X$.

(c) The expression $C$-psedudomonotone set-valued mapping in the framework of variational inequalities is due to Inoan and Kolumban [7].

Let $X$ be a topological vector space, $K$ be a nonempty convex subset of $X$, and $X^{*}$ the topological dual of $X$. A set-valued mapping $T: K \rightarrow 2^{X^{*}}$ is called 0 -segmentary closed ( $C$-pseudomonotone in the sense of Definition 2.3) if the function $h: K \times K \rightarrow \mathbb{R}$ defined by

$$
h(x, y)=-\sup _{y^{*} \in T(y)}\left\langle y^{*}, x-y\right\rangle
$$


is 0 -segmentary closed (see [5, 7]). In [7], the authors obtained some examples of this kind of mapping.

Definition 2.5. The set-valued mapping $T$ is called upper semi-continuous at $x \in X$ if for each open set $V$ containing $T(x)$ there is an open set $U$ containing $x$ such that for each $t \in U, T(t) \subseteq V$. We say that $T$ is upper semi-continuous on $X$ if it is upper semi-continuous at all $x \in X$.

LEMMA 2.6 (Tan [12]). Assume that for any $x \in X, T(x)$ is compact. Then $T$ is upper semi-continuous on $X$ if and only if for any net $\left\{x_{\alpha}\right\} \subset X$ such that $x_{\alpha} \rightarrow x$ and for every $y_{\alpha} \in T\left(x_{\alpha}\right)$, there exist $y \in T(x)$ and a subnet $\left\{y_{\beta}\right\}$ of $\left\{y_{\alpha}\right\}$ such that $y_{\beta} \rightarrow y$.

Definition 2.7 (Zhou and Tian [13]). Let $X$ be a nonempty set, $Y$ a topological space, and $T: X \rightarrow 2^{Y}$ a set-valued map. Then, $T$ is called transfer closed-valued if, for every $x, y \in K$ and $y \notin T(x)$, there exists $x^{\prime} \in X$ such that $y \notin \mathrm{cl} T\left(x^{\prime}\right)$, where cl $T\left(x^{\prime}\right)$ denotes the topological closure of $T\left(x^{\prime}\right)$.

REMARK 2.8. It is clear that, $T: X \rightarrow 2^{Y}$ is transfer closed-valued if and only if

$$
\bigcap_{x \in X} T(x)=\bigcap_{x \in X} \operatorname{cl} T(x) .
$$

Theorem 7 of [7] shows that the mapping $T: X \rightarrow 2^{Y}$ is $C$-pseudomonotone if and only if, for each $x, y \in X$,

$$
\operatorname{cl}\left(\bigcap_{z \in[x, y]} F(z)\right) \cap[x, y]=\left(\bigcap_{z \in[x, y]} F(z)\right) \cap[x, y],
$$

where $F(z)=\left\{x \in X \mid \sup _{f \in T(x)}\langle z-x, f\rangle \geq 0\right\}$.

From the inclusion

$$
\operatorname{cl}_{X}\left(\bigcap_{z \in[x, y]} F(z)\right) \cap[x, y] \subseteq \bigcap_{z \in[x, y]} \operatorname{cl}_{X}(F(z)) \cap[x, y],
$$

and the relation (2.1) through Remark 2.8 we infer that if for each $x, y \in K$ the restriction $F$ to the set $[x, y]$, that is $\left.F\right|_{[x, y]}:[x, y] \rightarrow 2^{X}$ defined by

$$
\left.F\right|_{[x, y]}(z)=\left\{w \in X: \sup _{f \in T(w)}\langle z-w, f\rangle \geq 0\right\}
$$

is a transfer-closed valued mapping, then $T$ is $C$-pseudomonotone.

The following theorem improves [7, Theorem 9].

THEOREM 2.9. Let $S: X \times X \rightarrow 2^{Y}$ be a set-valued mapping. Let $T: X \rightarrow 2^{Y}$ be defined by $T(x)=S(x, x)$. If $\left.S\right|_{\triangle \times \Delta}$ (the restriction of $S$ to the set $\Delta \times \triangle=$ $\{(x, x): x \in X\})$ is upper semi-continuous with nonempty compact values, then $T$ is $C$-pseudomonotone.

Proof. Let $x, y \in X,\left\{x_{i}\right\}$ in $X$ be such that $x_{i} \rightarrow x$ and

$$
\sup _{f \in S\left(x_{i}, x_{i}\right)}\left\langle(1-t) x+t y-x_{i}, f\right\rangle \geq 0 \quad \forall t \in[0,1], \forall i \in I .
$$


Since $\left.S\right|_{\triangle \times \triangle}$ has nonempty compact values and the bilinear mapping $\langle\cdot, \cdot\rangle$ is continuous then, for each $i$ and for $t=1$, there is $f_{i} \in S\left(x_{i}, x_{i}\right)$ such that

$$
\left\langle y-x_{i}, f_{i}\right\rangle=\sup _{f \in S\left(x_{i}, x_{i}\right)}\left\langle y-x_{i}, f\right\rangle .
$$

Now by applying Lemma 2.6 there exist a subnet $\left\{f_{j}\right\}$ of $\left\{f_{i}\right\}$ and $g \in T(x)=$ $S(x, x)$ such that $f_{j} \rightarrow g$. Hence, the continuity of the bilinear mapping $\langle\cdot, \cdot\rangle,(2.3)$, and (2.2) imply that $\langle y-x, g\rangle \geq 0$. Consequently $\sup _{f \in T(x)}\langle y-x, f\rangle \geq 0$. This completes the proof.

The next result improves [7, Corollary 11].

COROLlary 2.10. If $T: X \rightarrow 2^{Y}$ is an upper semi-continuous set-valued mapping with nonempty compact values, then $T$ is $C$-pseudomonotone.

PROOF. Let $S(x, y)=T(x)$ and apply Theorem 2.9.

For the next result we need the following lemma which is due to Blum and Oettli [1]. LEMMA 2.11. Let $D$ be a convex, compact set and let $K$ be a convex set. Let $f: D \times K \rightarrow \mathbb{R}$ be concave and upper semi-continuous in the first variable, and convex in the second variable. Assume that

$$
\max _{x \in D} f(x, y) \geq 0 \quad \forall y \in K .
$$

Then there exists $\bar{x} \in D$ such that $f(\bar{x}, y) \geq 0$ for all $y \in K$.

Now we have the following generalization of [7, Theorem 13] in the setting of topological vector spaces.

THEOREM 2.12. If:

(a) $T$ is B-pseudomonotone;

(b) $T(x)$ is nonempty convex and compact for each $x \in K$;

then $T$ is $C$-pseudomonotone.

Proof. Let $x, y \in X$ and $\left\{x_{i}\right\}$ be a net in $X$ with $x_{i} \rightarrow x$. Assume that

$$
\sup _{f \in T\left(x_{i}\right)}\left\langle(1-t) x+t y-x_{i}, f\right\rangle \geq 0 \quad \forall t \in[0,1], i \in I .
$$

Since the values of $T$ are compact, then for $t=0$ and for any $i \in I$, there exists $f_{i} \in T\left(x_{i}\right)$ such that

$$
\left\langle y-x_{i}, f_{i}\right\rangle=\sup _{f \in T\left(x_{i}\right)}\left\langle y-x_{i}, f\right\rangle \geq 0 .
$$

Hence, $\lim \inf _{i}\left\langle x-x_{i}, f_{i}\right\rangle \geq 0$, and so by condition (a), there exists $f(y) \in T(x)$ so that

$$
0 \leq \lim \sup \left\langle y-x_{i}, f_{i}\right\rangle \leq\langle y-x, f(y)\rangle
$$

(note that the inequality follows from (2.4) when $t=1$ ).

Therefore, the conclusion follows from (2.5). 


\section{Application}

Our aim in this section is to establish an existence result for the following problem which is called a variational inequality problem in the setting of topological vector spaces.

Let $X$ and $Y$ be two Hausdorff topological vector spaces, let $K$ be a nonempty, convex subset of $X$, and $T: K \rightarrow 2^{Y}$ be a set-valued mapping.

$$
\text { Find } x \in K \text { such that } \sup _{f \in T(x)}\langle y-x, f\rangle \geq 0 \quad \forall y \in K \text {. }
$$

Such variational inequalities occur, for example, in the study of optimality for parametric variational problems of the form

$$
\min _{u \in K} \max _{\lambda \in \Lambda} \int_{\Omega} G(\lambda, t, u(t), \nabla u(t)) d t,
$$

where $K$ is a closed and convex set of the Sobolev space $H^{1}(\Omega), \Lambda$ is a set of parameters and $\Omega$ is a bounded subset of $\mathbb{R}^{n}$ (see for instance [7, 11]).

We denote the solution set of (VI) by $S$. The next theorem is needed later.

By checking the proof of Theorem 2.1 in [3, Page 113, lines 15-19], one can realize that the authors, in fact, obtained the following generalization of the Fan-KnasterKuratowski-Mazurkiewicz lemma [4].

THEOREM 3.1. Let $X$ be a topological vector space and $K$ be a nonempty convex subset of $X$. Suppose that $\Gamma, \widehat{\Gamma}: K \rightarrow 2^{K}$ are two multivalued mappings such that:

(i) $\widehat{\Gamma}(x) \subseteq \Gamma(x)$, for all $x \in K$;

(ii) $\widehat{\Gamma}$ is a Knaster-Kuratowski-Mazurkiewicz (KKM) map;

(iii) for each finite subset $A$ of $K, \Gamma$ is transfer closed-valued on co $A$;

(iv) for each $x, y \in K, \operatorname{cl}_{K}\left(\bigcap_{z \in[x, y]} \Gamma(z)\right) \cap[x, y]=\left(\bigcap_{z \in[x, y]} \Gamma(z)\right) \cap[x, y]$;

(v) there is a nonempty compact convex set $B \subseteq K$ such that $\operatorname{cl}_{K}\left(\bigcap_{x \in B} \Gamma(x)\right)$ is compact.

Then, $\bigcap_{x \in K} \Gamma(x) \neq \emptyset$.

We remark that condition (iv) of Theorem 3.1 is equivalent to the $C$-pseudomonotonicity of the set-valued mapping $T: X \rightarrow 2^{Y}$ (see [7, Theorem 7]).

THEOREM 3.2. Let $X$ and $Y$ be two Hausdorff topological vector spaces, $K a$ nonempty convex subset of $X$, and $T: K \rightarrow 2^{Y}$ be a set-valued mapping. Assume that:

(a) $T$ is $C$-pseudomonotone;

(b) for each finite subset $A$ of $K, T$ is upper semi-continuous on $\operatorname{co} A$;

(c) there exist a compact, convex subset $B \subseteq K$ and a compact set $D \subseteq K$ such that

$$
\forall x \in K \backslash D \text { and } \exists y \in B \quad \text { such that } \sup _{f \in T(x)}\langle y-x, f\rangle<0 ; \quad \text { and }
$$

(d) $T(x)$ is nonempty compact, for all $x \in K$.

Then the solution set of (VI) is a nonempty, compact subset of $B$. 
Proof. Define $\Gamma=\hat{\Gamma}: K \rightarrow 2^{K}$ by

$$
\Gamma(y)=\left\{x \in K: \sup _{f \in T(x)}\langle y-x, f\rangle \geq 0\right\} .
$$

We now show that $\Gamma$ fulfils all of the assumptions of Theorem 3.1. Note that for each $y \in K, \Gamma(y) \neq \varnothing$ since $y \in \Gamma(y)$. We first show that $\Gamma$ is a $\mathrm{KKM}$ map. Assume on the contrary that there exist $A=\left\{x_{1}, x_{2}, \ldots, x_{n}\right\} \subseteq K, z \in \operatorname{co} A$ and $z \notin \bigcup_{i \in\{1,2, \ldots, n\}} \Gamma\left(x_{i}\right)$. Then, by the definition of $\Gamma$ and choosing a fixed element $f \in T(z)$, we have

$$
\left\langle x_{i}-z, f\right\rangle<0 \quad \forall i=1, \ldots, n .
$$

This and $z \in \operatorname{co} A$ imply that $0=\langle z-z, f\rangle<0$ which is a contradiction. Now we prove that for each finite subset $A$ of $K, \Gamma$ is transfer closed-valued on co $A$. To see this, applying Remark 2.8 , we must show the following equality

$$
\bigcap_{y \in \operatorname{co} A} \operatorname{cl}(\Gamma(y) \cap \operatorname{co} A)=\bigcap_{y \in \operatorname{co} A}(\Gamma(y) \cap \operatorname{co} A),
$$

for each finite subset $A$ of $K$.

Let $z \in \bigcap_{y \in \operatorname{co} A} \operatorname{cl}(\Gamma(y) \cap \operatorname{co} A)$. Hence, for each $y \in \operatorname{co} A$, there exists a net $\left\{x_{i}\right\}$ in $\Gamma(y) \cap \operatorname{co} A$ which converges to $z$. Thus, for all $i$,

$$
\sup _{f \in T\left(x_{i}\right)}\left\langle y-x_{i}, f\right\rangle \geq 0
$$

Since, by condition (d), $T\left(x_{i}\right)$ is compact, for all $i$, and the bilinear mapping $\langle\cdot, \cdot\rangle$ is continuous, there exists $f_{i} \in T\left(x_{i}\right)$ such that

$$
\left\langle y-x_{i}, f_{i}\right\rangle=\sup _{f \in T\left(x_{i}\right)}\left\langle y-x_{i}, f\right\rangle \geq 0
$$

Now by Lemma 2.6, there exist a subnet $\left\{f_{j}\right\}$ of $\left\{f_{i}\right\}$ and a $g \in T(z)$ such that $f_{j} \rightarrow g$. Hence, from (3.1) we obtain $\langle y-z, g\rangle \geq 0$, and so $\sup _{f \in T(x)}\langle y-z, f\rangle \geq 0$. This shows that $z \in \Gamma(y)$. Since $x_{i} \rightarrow z, x_{i} \in \operatorname{co} A$, and co $A$ is compact (note that $A$ is a finite subset of $K$ ) we have $z \in \operatorname{co} A$. Consequently, $z \in \Gamma(y) \cap \operatorname{co} A$. Therefore,

$$
\bigcap_{y \in \operatorname{co} A} \operatorname{cl}(\Gamma(y) \cap \operatorname{co} A) \subseteq \bigcap_{y \in \operatorname{co} A}(\Gamma(y) \cap \operatorname{co} A) .
$$

The reverse of the above inclusion is obvious. From condition (a) and [7, Theorem 7] we obtain the following expression for each $x, y \in K$ :

$$
\operatorname{cl}_{K}\left(\bigcap_{z \in[x, y]} \Gamma(z)\right) \cap[x, y]=\left(\bigcap_{z \in[x, y]} \Gamma(z)\right) \cap[x, y] .
$$


From condition (d) we have that $\mathrm{cl}_{K}\left(\bigcap_{x \in B} \Gamma(x)\right)$ is compact. Therefore, $\Gamma$ satisfies conditions (i)-(v) of Theorem 3.1 and so there exists $\bar{x} \in K$ such that $\bar{x} \in \bigcap_{y \in K} \Gamma(y)$. Hence, we have

$$
\sup _{f \in T(\bar{x})}\langle y-\bar{x}, f\rangle \geq 0 \quad \forall y \in K .
$$

Thus, $\bar{x}$ is a solution of (VI), that is $\bar{x} \in S$, where $S$ denotes the solution set of (VI), and hence $S$ is nonempty. From condition (c) we deduce that $S$ is a subset of $B$ and so $S=S \cap B$. Now since $B$ is compact and $S=S \cap B$ we obtain that $S$ is a compact subset of $B$ with respect to the induced topology from $X$ on $S$. This completes the proof.

REMARK 3.3. Let $X$ be a normed space and let $X^{*}$ be the topological dual of $X$. Then the coercivity condition (c) in Theorem 3.2 is weaker than the following condition of [7, Theorem 15]:

there exists a weakly compact subset $A \subseteq K$ and $z_{0} \in K$ such that

$$
\sup _{f \in T(x)}\left\langle z_{0}-x, f\right\rangle<0 \quad \text { for every } y \in K \backslash A \text {. }
$$

Indeed, let $A$ be a weakly compact subset $A \subseteq K$. Then by the Mazur theorem, $\overline{\operatorname{co} A}$ is compact and convex. Let $B=\overline{\operatorname{co} A}$ and $D=\left\{z_{0}\right\}$. Since $K \backslash \overline{\operatorname{co} A} \subseteq K \backslash A$, then from the above condition we obtain condition (c) in Theorem 3.2.

One can see that condition (c) of [7, Theorem 15] is a particular case of condition (b) of Theorem 3.2. Hence, Theorem 3.2 generalizes and improves [7, Theorem 15].

The following corollary improves [7, Corollary 16].

Corollary 3.4. Let $X$ and $Y$ be two Hausdorff topological vector spaces, let $K$ be a nonempty convex subset of $X$, and let $T: K \rightarrow 2^{Y}$ be a set-valued mapping. Let $\bar{x}$ be a solution of (VI). Assume that $T(\bar{x})$ is a compact, convex set. Then there exists $\bar{f} \in T(\bar{x})$ such that

$$
\langle y-\bar{x}, \bar{f}\rangle \geq 0 \quad \forall y \in K .
$$

Proof. Define $P: T(\bar{x}) \times K \rightarrow \mathbb{R}$ by $P(f, y)=\langle y-\bar{x}, f\rangle$, and put $D=T(\bar{x})$. It is clear that the mapping $P$ is concave and upper semi-continuous in the first variable, and convex in the second variable. Now since $\bar{x}$ is a solution of (VI) and $T(\bar{x})$ is compact we have

$$
\max _{f \in T(\bar{x})} P(f, y)=\max _{f \in T(\bar{x})}\langle y-\bar{x}, f\rangle=\sup _{f \in T(\bar{x})}\langle y-\bar{x}, f\rangle \geq 0 .
$$

Consequently $P$ satisfies all of the assumptions of Lemma 2.11 and so there exists $\bar{f} \in T(\bar{x})$ such that

$$
P(\bar{f}, y)=\langle y-\bar{x}, \bar{f}\rangle \geq 0 \quad \forall y \in K
$$




\section{Acknowledgements}

The author would like to thank a referee for valuable suggestions and remarks. The author was in part supported by the School of Mathematics, Institute for Research in Fundamental Sciences (IPM), P.O. Box 19395-5746, Tehran, Iran (research code 87490015).

\section{References}

[1] E. Blum and W. Oettli, "From optimization and variational inequalities to equilibrium problems", Math. Stud. 63 (1994) 123-145.

[2] H. Brezis, "Équations et inéquations non lineáires dans les espaces vectoriels en dualité", Ann. Inst. Fourier (Grenoble) 18 (1968) 115-175.

[3] M. Fakhar and J. Zafarani, "Generalized vector equilibrium problems for pseudomonotone multivalued bifunctions", J. Optim. Theory Appl. 126 (2005) 109-124.

[4] K. Fan, "Some properties of convex sets related to fixed point theorems", Math. Ann. 266 (1984) 519-537.

[5] A. P. Farajzadeh and J. Zafarani, Equilibrium problems and variational inequalities in topological vector spaces, Optimization, DOI: 10.1080/02331930801951090, (2008).

[6] N. Hadjisavvas and S. Schaible, "Generalized monotone maps", in Handbook of generalized convexity and generalized monotonicity (eds N. Hadjisavvas, S. Komlosi and S. Schaible), (Springer, Berlin, 2005) 389-420.

[7] D. Inoan and J. Kolumban, "On pseudomonotone set-valued mappings", Nonlinear Anal. 68 (2008) 47-53.

[8] S. Karamardian, "Complementarity problems over cones with monotone and pseudomonotone maps", J. Optim. Theory Appl. 18 (1976) 445-454.

[9] S. Komlosi, "Generalized monotonicity in nonsmooth analysis", in Generalized convexity (eds S. Komlosi, T. Rapcsak and S. Schaible), (Springer, Heidelberg, 1994) 263-275.

[10] P. D. Panagiotopoulos, "Hemivariational inequalities", in Applications in mechanics and engineering (Springer, Berlin, 1993).

[11] B. N. Pshenichnyï, "Necessary condition for an extremum", in Pure and applied mathematics, Volume 4 (ed. L. W. Neustade), (Marcel Dekker, New York, 1971) (translated from the Russian by K. Makowski).

[12] N. T. Tan, "Quasi-variational inequalities in topological linear locally convex Hausdorff spaces", Math. Nachr. 122 (1985) 231-245.

[13] J. Zhou and G. Tian, "Transfer method for characterizing the existence of maximal elements of binary relations on compact or non compact sets", SIAM J. Optim. 2 (1992) 360-375. 